\title{
EDITORIAL
}

\section{La nueva actualidad de la salud mental}

La salud mental y la atención a los trastornos mentales han ocupado un lugar destacado en el debate social en diversos momentos históricos. Así, por ejemplo, la introducción del psicoanálisis supuso una auténtica conmoción en los inicios del siglo veinte, las aportaciones de los culturalistas fueron best sellers en los años cincuenta y la voluntad de descifrar el tipo de cuestionamiento de los usos sociales que encerraba la locura lo fue en los sesenta y setenta de la mano de los llamados antipsiquiatras, de los reformadores de la psiquiatría o de Michel Foucault y seguidores ya en los sesenta y setenta.

En los años ochenta las referencias a la salud o los trastornos mentales fuera de los ámbitos especializados pasaron a ser meramente marginales. A la sombra de las grandes revoluciones conservadoras, la atención a la salud mental dejó de ser considerada un desafío para el Estado del Bienestar o una fuente de inspiración para el pensamiento crítico, para pasar a ser observada únicamente como un potencial mercado en el que la industria podría realizar beneficios.

El pensamiento psiquiátrico y la actividad de los psiquiatras pasaron a supeditarse de un modo absoluto a este fin. La salud mental dejó de ser pensada como un logro difícilmente construido, y pasó a considerarse un estado natural sólo amenazado por alteraciones bioquímicas del funcionamiento cerebral, pues se esperaba que el desarrollo paralelo de las neurociencias -que hay que decir que se ha producido a pesar de los psiquiatras y no gracias a ellos- pudiera explicar las enfermedades. Los psiquiatras pasamos a ser prescriptores y, en todo caso, testigos y voceros de las bondades de los remedios que se disputaban el nuevo mercado. Nuestro gran desafío teórico pasó a ser la construcción de grandes sistemas ateóricos (como el DSM) que permitieran identificar los trastornos sobre los que se supone que cada uno de los remedios puede actuar más específicamente.

Aunque la perspectiva instaurada en los ochenta siga siendo hegemónica y permanezca incuestionada en los órganos de expresión de la comunidad psiquiátrica y, por supuesto, en las instituciones académicas, lo cierto es que hoy tenemos datos suficientes para sostener que ha resultado un fracaso. Los remedios que se suponía que iban a ser cada vez más específicos para trastornos cada vez más precisamente definidos, han resultado ser todo menos específicos. Recuérdese que los inhibidores selectivos de la recaptación de la serotonina (ISRS ) pretendían haberse convertido en la «bala de plata» que actuaba contra lo que se suponía que era la alteración específica de la depresión, frente a la inespecificidad de los antiguos -y tan baratos- antidepresivos tricíclicos. Incluso se crearon categorías diagnósticas nuevas -la depresión atípica- para excluir los trastornos sufridos por algunos pacientes que no respondían bien a los nuevos remedios. Hoy, los ISRS son el tra- 
tamiento farmacológico de primera elección de la depresión, pero también del trastorno de angustia, de la ansiedad generalizada, del trastorno obsesivo compulsivo, de los trastornos de la personalidad, de los trastornos del control de impulsos y de otros muchos. Si tenemos en cuenta, a la vez, que a los antipsicóticos responden los síntomas positivos de los pacientes esquizofrénicos, los delirios crónicos, los cuadros maníacos, los síntomas psicóticos de los trastornos mentales orgánicos y otros, quizás podíamos pensar que, aunque sólo fuera en consideración de lo que podemos aprender sobre nuestro trabajo como clínicos prescriptores -necesitaríamos articular nuestras clasificaciones- o, mucho mejor, pensar sobre la salud mental y los trastornos mentales sobre nuevas bases.

En los últimos años se han producido algunas señales de que existe una nueva preocupación social por la salud mental y sus alteraciones al menos en lo que solemos llamar el mundo desarrollado. Sin hacer mención a la proliferación de instrumentos de autoayuda que pretenden responder a la necesidad subjetivamente experimentada por multitudes de preservar su salud mental, si atendemos sólo a las manifestaciones institucionales encontramos que la salud y los trastornos mentales han vuelto a ser motivo de preocupación política, al menos en Europa.

La Organización Mundial de la Salud nos ha provisto, a través del proyecto ATLAS, de una visión de conjunto que nos permite conocer la situación a grandes rasgos de la atención a la salud mental en todo el mundo.

La Oficina Regional Europea de la Organización Mundial de la Salud propició el acuerdo entre veinticinco ministros de sanidad europeos, materializado en los llamados acuerdos de Helsinki, para tomar medidas concretas tendentes a mejorar la salud mental y actuar contra sus alteraciones en todos ellos. La Comisión Europea, a su vez, ha promovido la redacción del llamado Libro Verde, que reúne recomendaciones para articular una política de salud mental conjunta en Europa. El Consejo de Europa ha desarrollado importantes documentos y trabaja en el desarrollo de nuevos instrumentos que pretenden servir para proteger los derechos de las personas con trastornos mentales.

Algunos gobiernos, como el británico, han incrementado los fondos dedicados a la atención a la salud mental de un modo muy significativo, poniendo en marcha programas por los que han visto la luz, además de los importantes recursos ya existentes, los equipos de tratamiento asertivo comunitario, los equipos de atención en crisis o los equipos de atención temprana. El 31 de julio, el ministro de sanidad de ese país anunciaba el funcionamiento de los primeros equipos del plan por el que el Servicio Nacional de Salud va a dotarse de los psicoterapeutas necesarios para ofrecer psicoterapia como tratamiento de rutina para pacientes con ansiedad o depresión. Y la prestigiosa revista médica The Lancet, ha dedicado una serie de artículos haciéndose eco de todo lo anterior y proponiendo vías de actua- 
EDITORIAL

ción a través de una serie de artículos redactados por un llamado Lancet Global Mental Health Group, que reúne a 38 expertos internacionales en el tema.

A la vista de estos informes (alguno de los cuales utiliza datos relativamente antiguos), España se encuentra entre los países europeos que menos recursos dedica a la atención a la salud mental. La estructura del Estado de las Autonomías, con la circunstancia de que la mayor parte de las competencias sanitarias están transferidas, hace que no sea fácil trasponer las directivas europeas. Sin embargo, muy recientemente se han producido algunos avances importantes propiciados desde el gobierno central. En primer lugar se ha regulado por ley una cartera de servicios del sistema nacional de salud, que obliga a las autonomías y que define las responsabilidades en la materia de la atención primaria de salud y la atención especializada. Dicha cartera incluye los tratamientos farmacológicos, la psicoterapia y la rehabilitación, lo que, hasta ese momento, era objeto de discusión.

Además, a iniciativa del Ministerio, el Consejo Interterritorial ha aprobado la Estrategia de Salud Mental del Sistema Nacional de Salud. Se trata de un documento que es, necesariamente, un documento de mínimos, porque expresa un acuerdo de todas las comunidades autónomas (un acuerdo, sorprendente en los tiempos que corren, y por ello, una buena noticia). Pero es un documento suficiente que, sin duda, servirá para armonizar el desarrollo de los servicios autonómicos y mejorar su calidad.

Una de las constantes en los documentos europeos es la constatación de que la salud mental, globalmente considerada, no puede ser una tarea sólo sanitaria sino que requiere de un trabajo coordinado en el que deben participar muchos otros departamentos. El documento titulado «Modelo de Atención en el Sistema para la Autonomía del Enfermo Mental Grave» publicado por el IMSERSO y fruto también de un amplio consenso, abre el camino a esta perspectiva, que debería extenderse necesariamente a otros departamentos, con especial urgencia a los responsables de las prisiones y la justicia, donde se dan situaciones inaceptables.

Este resurgir del tema no está exento de peligros. A los profesionales de la salud mental nos toca promover el debate social que permita sortear alguno de ellos: la delimitación del objeto de nuestro trabajo como clínicos (los trastornos mentales, ¿definidos con qué criterio?) del malestar general y de los comportamientos antiéticos, los obstáculos sociales, culturales y normativos a la integración y recuperación de las personas que sufren trastornos mentales, el uso coercitivo de los instrumentos de la psiquiatría (véase el nuevo protocolo de repatriación de emigrantes), los nuevos modos de vulneración de los derechos de los enfermos mentales. Con toda seguridad, oiremos hablar de ellos. 